\title{
Matrix metalloproteinases in right ventricular failure
}

Marc A. Simon

I thank Dr Tanus-Santos for raising an important point (Tanus-Santos, J. E. Targeting RV failure and cardiomyocyte injury in acute pulmonary thromboembolism. Nat. Rev. Cardiol. doi:10.1038/ nrcardio.2013.12-c1 $)^{1}$ in response to $\mathrm{my}$ Review (Simon, M. A. Assessment and treatment of right ventricular failure. Nat. Rev. Cardiol. 10, 204-218 [2013]). ${ }^{2}$ Matrix metalloproteinases (MMPs) are likely to have a major role in the response to right ventricular injury, permitting ventricular dilation and remodelling downstream from a variety of agents that can adversely affect the right ventricle. Further studies are needed to characterize the specific MMPs concerned, as well as the pathways involved, such as inflammation, cell migration, collagen degradation, or apoptosis. This area of investigation is important, highlighting the need to better understand the pathophysiology of right ventricular failure and the exciting experimental work being conducted in this field.

Division of Cardiology, Department of Medicine, University of Pittsburgh, Heart \& Vascular Institute, University of Pittsburgh Medical Centre, Scaife Hall S-555, 200 Lothrop Street, Pittsburgh, PA 15213, USA.

simonma@upmc.edu

Competing interests

The author declares associations with the following companies and organizations: AHA, Pfizer, The Pittsburgh Foundation, and United Therapeutics. See the article online for full details of the relationships.

1. Tanus-Santos, J. E. Targeting RV failure and cardiomyocyte injury in acute pulmonary thromboembolism. Nat. Rev. Cardiol. http:// dx.doi.org/10.1038/nrcardio.2013.12-c1.

2. Simon, M. A. Assessment and treatment of right ventricular failure. Nat. Rev. Cardiol. 10, 204-218 (2013). 